\title{
Total nasal septal reconstruction using costal cartilage in difficult cases of secondary septoplasty
}

\author{
Kapil S. Agrawal, Raghav Shrotriya
}

Department of Plastic and Reconstructive Surgery, Seth GS Medical College and KEM Hospital, Mumbai 400012, India.

Correspondence to: Dr. Raghav Shrotriya, Department of Plastic and Reconstructive Surgery, Seth GS Medical College and KEM Hospital, Opp Ward 16, 2nd Floor, Gynec Wing, Mumbai 400012, India. E-mail: dr.raghav.s@gmail.com

How to cite this article: Agrawal KS, Shrotriya R. Total nasal septal reconstruction using costal cartilage in difficult cases of secondary septoplasty. Plast Aesthet Res 2016;3:306-10.

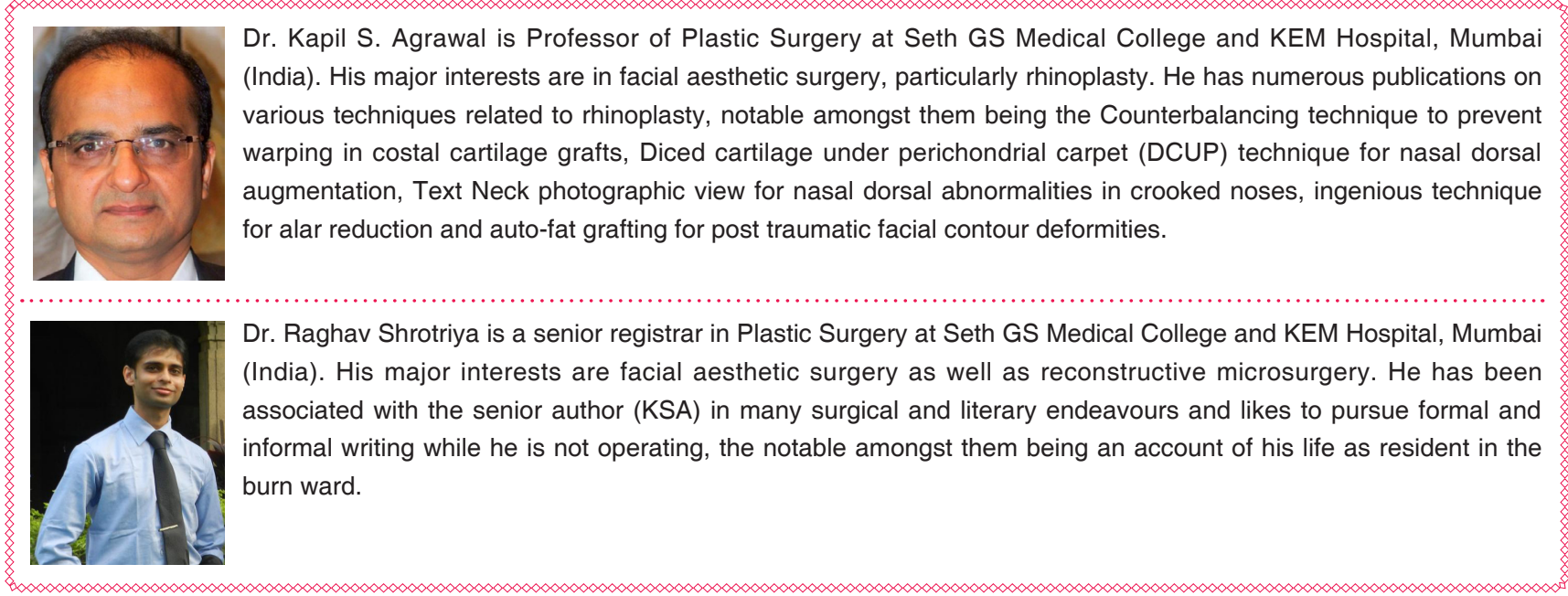

Article history:

Received: 02-03-2016

Accepted: 24-08-2016

Published: 20-09-2016

Key words:

Rhinoplasty,

nasal septum reconstruction,

costal cartilage graft

\section{ABSTRACT}

Rhinoplasty and septal reconstruction often require the use of cartilage grafts. Complete nasal septal reconstruction may be required in very specific situations like difficult secondary septoplasty or severely deformed post traumatic noses. Usually in these cases the septal cartilage or bone is insufficient for septal reconstruction. The authors hereby describe a new technique of complete septal reconstruction by using the 7th rib (costal) cartilage combined with author's ingenious technique to prevent warping.

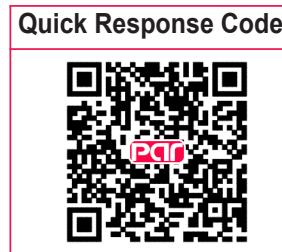




\section{INTRODUCTION}

Rhinoplasty and septal reconstruction often require the use of cartilage grafts. Occasionally, the unsuspecting rhinoplasty surgeon may hitherto stumble upon such noses which have paucity of native cartilaginous and bony septum. In post-septoplasty severely deviated nose and severely deformed post traumatic nose, the native septal cartilage and bony septum may be thin, fragmented or inadequate. The reconstruction of a complete septum with costal cartilage may restore a strong support in such situations. Here we describe two such cases.

\section{CASE REPORT}

\section{Case 1}

A 26-year-old male had a history of injury to the nose by cricket ball 5 years ago. His nose had gradually deviated and owing to severe breathing problems, he underwent septoplasty 2 years ago. There was considerable relief in breathing after the surgery but the nose remained crooked. He presented to us with a crooked nose for aesthetic correction [Figure $1 \mathrm{~A}$ and $\mathrm{B}$ ].

The patients were assessed postoperatively by clinical examination, photography, nasoendoscopy. The dorsal symmetry was confirmed postoperatively by "text neck photographic view". ${ }^{[1]}$ The first patient has been following up regularly since 18 months [Figure $1 \mathrm{C}$ and $\mathrm{D}$ ]. Clinically the nose and septum is straight and airway is patent as confirmed by nasoendoscopy [Figure 2].

\section{Case 2}

A 35-year-old female presented with severely deformed and scarred nose and breathing difficulty. Owing to a road accident one year ago, she had an adherent scar running from right nostril till junction of cartilaginous and bony dorsum, resulting in partition of right nostril. There was disjunction of cartilaginous dorsum and detachment
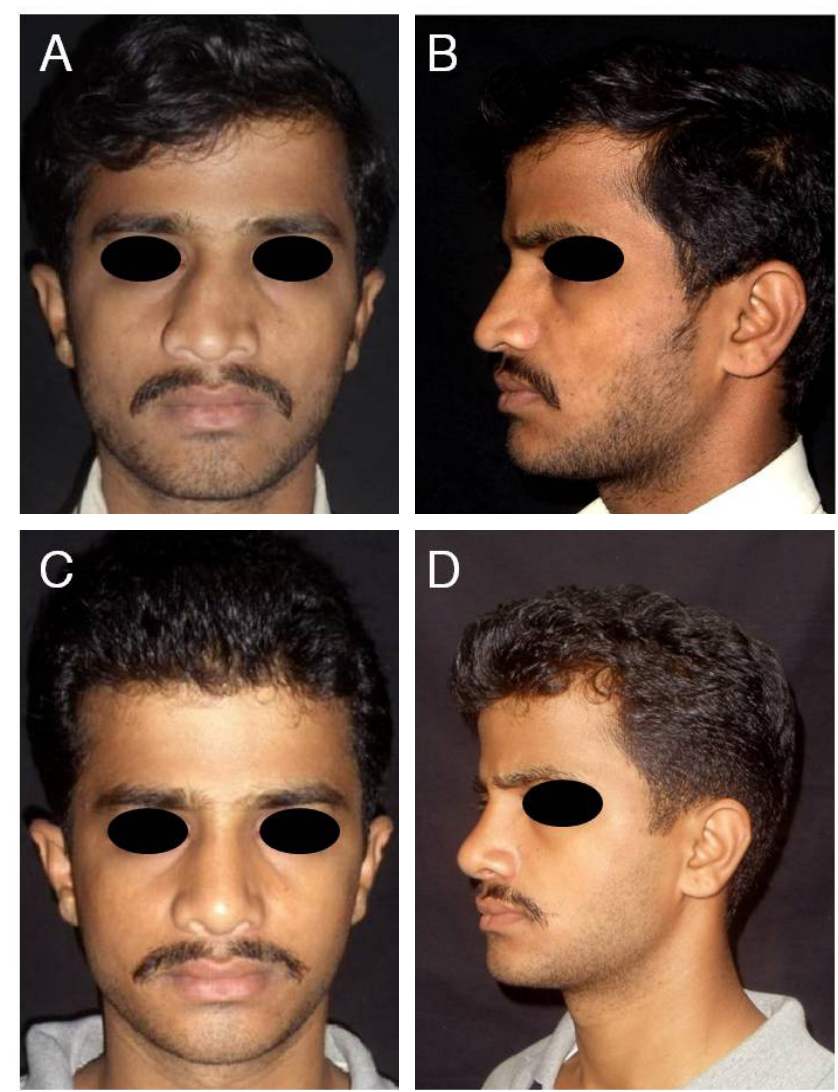

Figure 1: Patient 1. A: Preoperative photograph, frontal view; B: preoperative photograph, lateral view; C: the 1-year postoperative photograph, frontal view; D: the 1-year postoperative photograph, lateral view

of left medial canthus which was displaced in an oblique position. Also, there was shortage of skin between right ala and nasal tip [Figure $3 \mathrm{~A}$ and $\mathrm{B}]$.

The patient was operated in two stages. Initially she underwent left medial canthopexy, release of synechia of right nostril, adhesiolysis, scar revision, and insertion of a costal cartilage strut to the nasal dorsum, through the existing scar. The scar revision and insertion of a
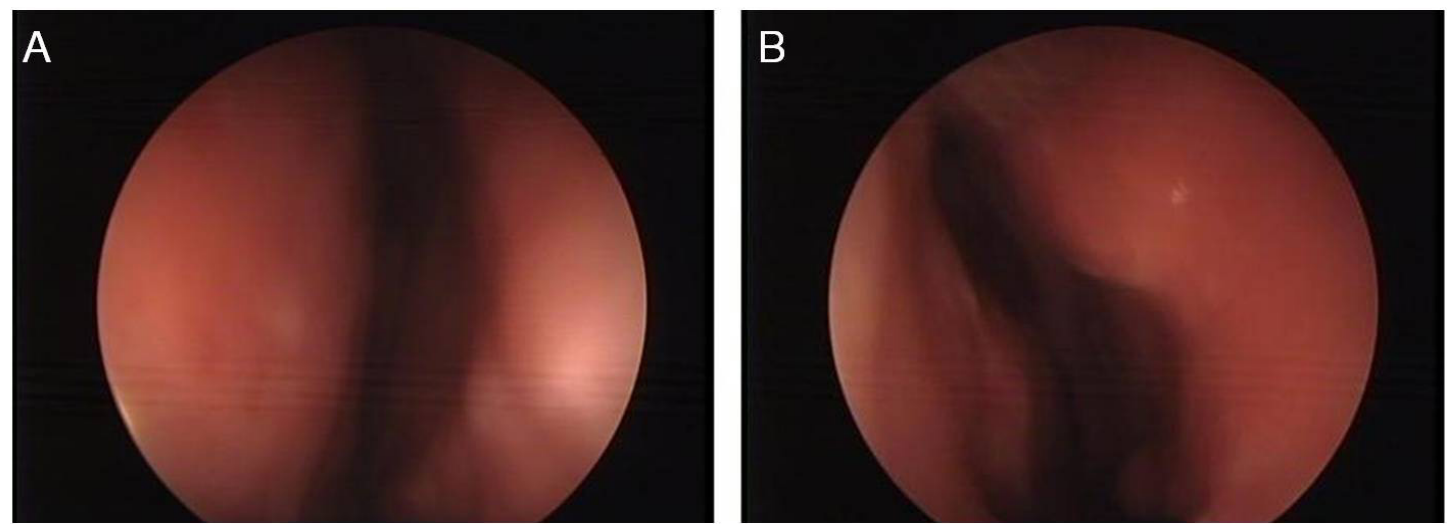

Figure 2: Patient 1. Postoperative naso-endoscopic view on right (A) and left (B) side showing patent airway 

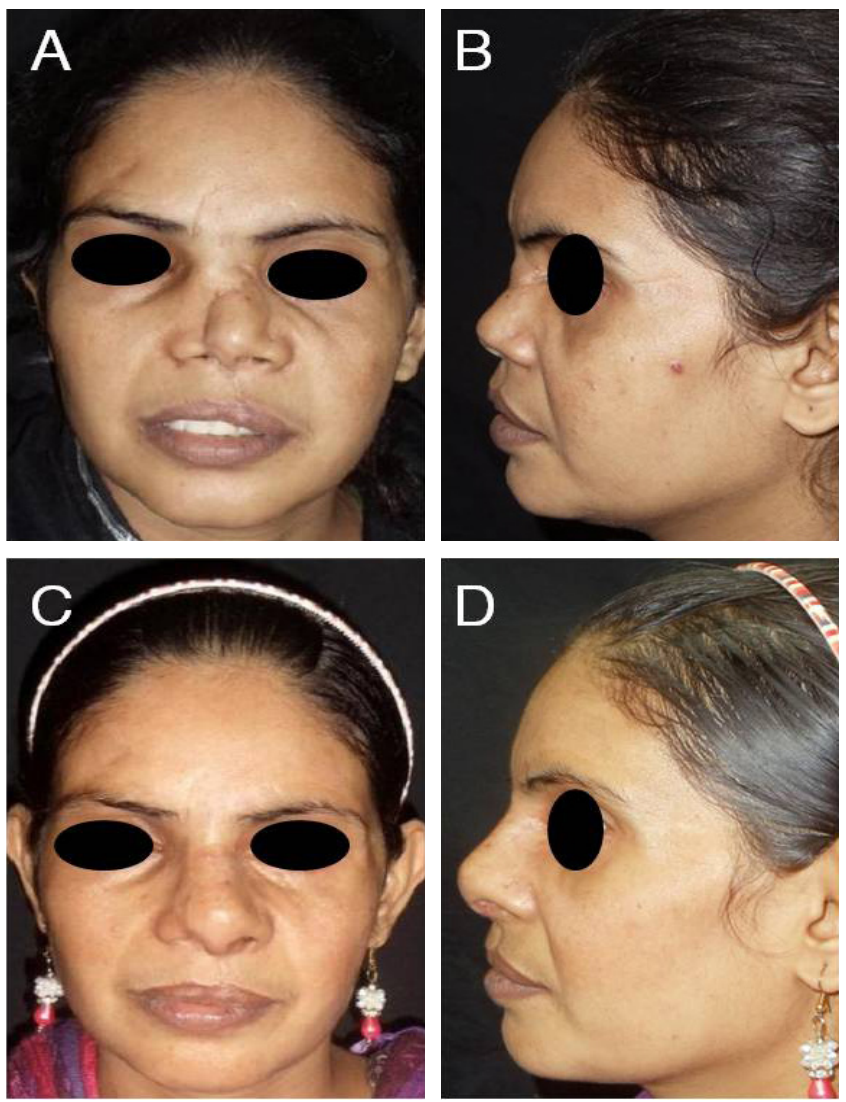

Figure 3: Patient 2. A: Preoperative photograph, frontal view; B: preoperative photograph, lateral view; C: the 3-month postoperative photograph, frontal view; D: the 3-month postoperative photograph, lateral view

strut not only improved the scar and stretched the skin envelope, but also prevented further adhesions and improved her breathing up to some extent. The final surgery was done one year later for reconstruction of the nasal septum.

The patient had clinically straight nose and patent airway on 3-month follow-up after which she was lost to follow-up [Figure $3 \mathrm{C}$ and $\mathrm{D}$.

\section{Surgical technique}

Being broad and flat usually, the 7th costal cartilage was used for both the patients [Figure 4A]. Careful and indulgent carving of cartilage was required to get 2 equal and thin plates. Any uneven surface or discrepancy in thickness of the 2 plates was further carved either with the help of a knife or a burr. Since these plates have a tendency to warp, a straight plate was formed using authors' Counterbalancing technique ${ }^{[2]}$ in which concave surfaces of both the plates are sutured together to get a strong and an absolutely straight plate [Figure 4B and C].

The authors first used 4-0 polyglactin as, due to its better knotting property and strength, it takes all the stress in conforming the 2 concave plates. This allowed the use of a thinner non absorbable sutures (5-0 polypropylene), which usually break if used alone. Authors avoid using 4-0 polypropylene alone as it is too thick and the knots usually get exposed through the septal muco-perichondrial flaps. The length of the plate was dictated by the dimensions of the native septum as measured from the distal end of the nasal bones to the anterior nasal spine. The thickness (width) of the neoseptum was around $3 \mathrm{~mm}$ in both cases.

The neo-septum was kept $5-7 \mathrm{~mm}$ longer on its proximal end which was fed into the groove created in the middle of nasal bone for adequate support. Bilateral spreader grafts were sutured to the neo-septum, which was fixed in place, proximally to nasal bones and distally to the anterior nasal spine, by drilling 2 holes on each side [Figure 5]. The fixation was done with 4-0 polypropylene sutures. The neo-septum rests comfortably on the vomer bone. The rest of the steps were same as in any extracorporeal septorhinoplasty case.

\section{DISCUSSION}

Septal reconstruction is a frequently required difficult
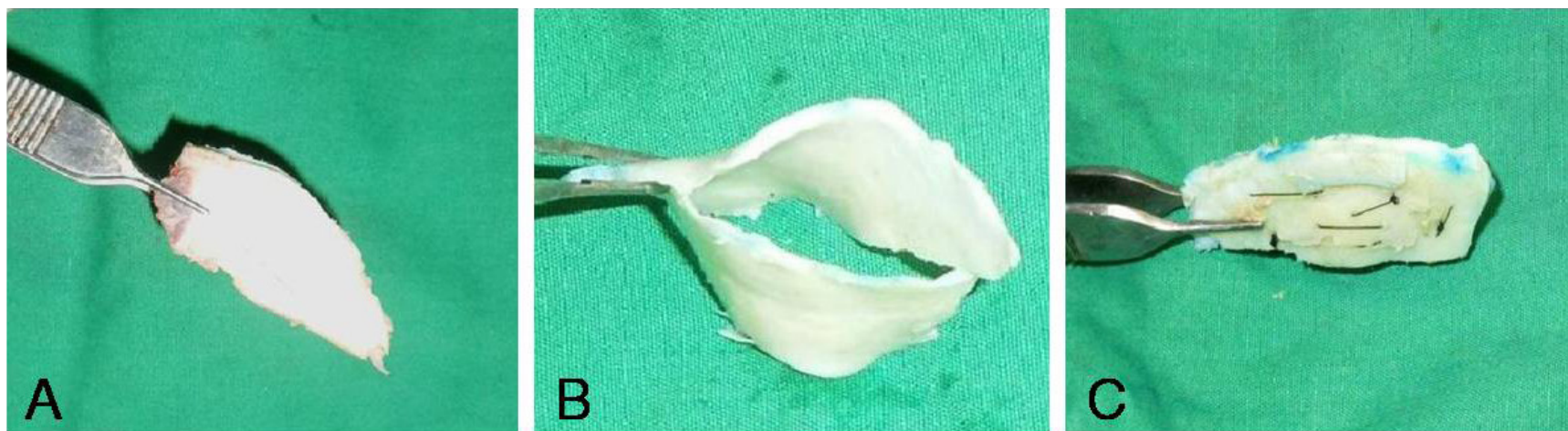

Figure 4: A: the 7th costal cartilage graft; B: warping after carving of cartilage pieces; C: prepared neoseptum using counterbalancing technique to control warping 


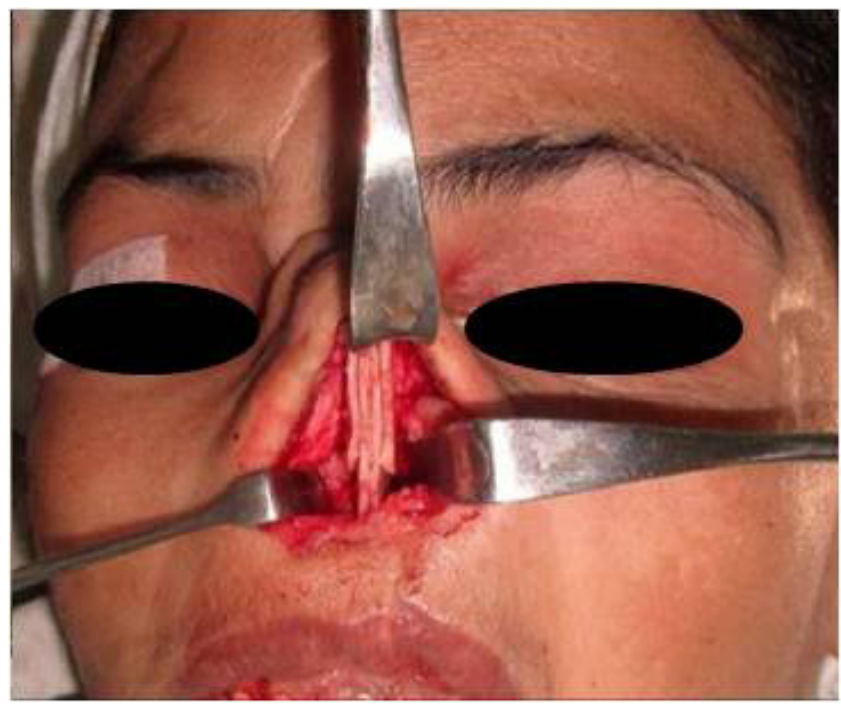

Figure 5: Placement of neoseptum

step in post septoplasty or post traumatic rhinoplasty cases. King et al. $^{\left[{ }^{[3]}\right.}$ first described extracorporeal septoplasty in 1952 which generally produces good results. In this procedure, popularized by Gubisch in the early 1980s, the cartilaginous and bony septum were removed intact, redundant cartilage and fracture lines were excised, and the remaining pieces were sutured together ${ }^{[4,5]}$ The main indication for extracorporeal septoplasty is severely deviated crooked septum causing both functional as well as cosmetic deformity. In majority of cases, the deformed native septum provides enough cartilage or bony plate by which a strong neoseptum can be rebuilt. L-strut [Figure 6A] or neo-septum can be made by either septal cartilage or the bony plate, or a combination thereof. ${ }^{[6]}$

An easier option to rebuild a neo-septum is polydioxanone sulfate (PDS) plate, on which small fragments can be sutured to act as a scaffold. ${ }^{[7-9]}$ In case of paucity of septal cartilage, conchal cartilage can also be used. In the first case, due to previous septoplasty, enough cartilage and bone could not be found to reconstruct a neo-septum. The second case was severe post traumatic nasal deformity. Here too, only fragments of cartilage and bony plate could be found, which could not be used to build a neo-septum.

Although PDS plate could have been used for the reconstruction but in the first case, the authors were caught unawares and had to resort to the technique being described. The success of first case encouraged the use of the technique in second case. The replacement of full cartilaginous plate which is resting over vomer bone or in the vomerine groove akin to the native septum, and fixed properly to the anterior
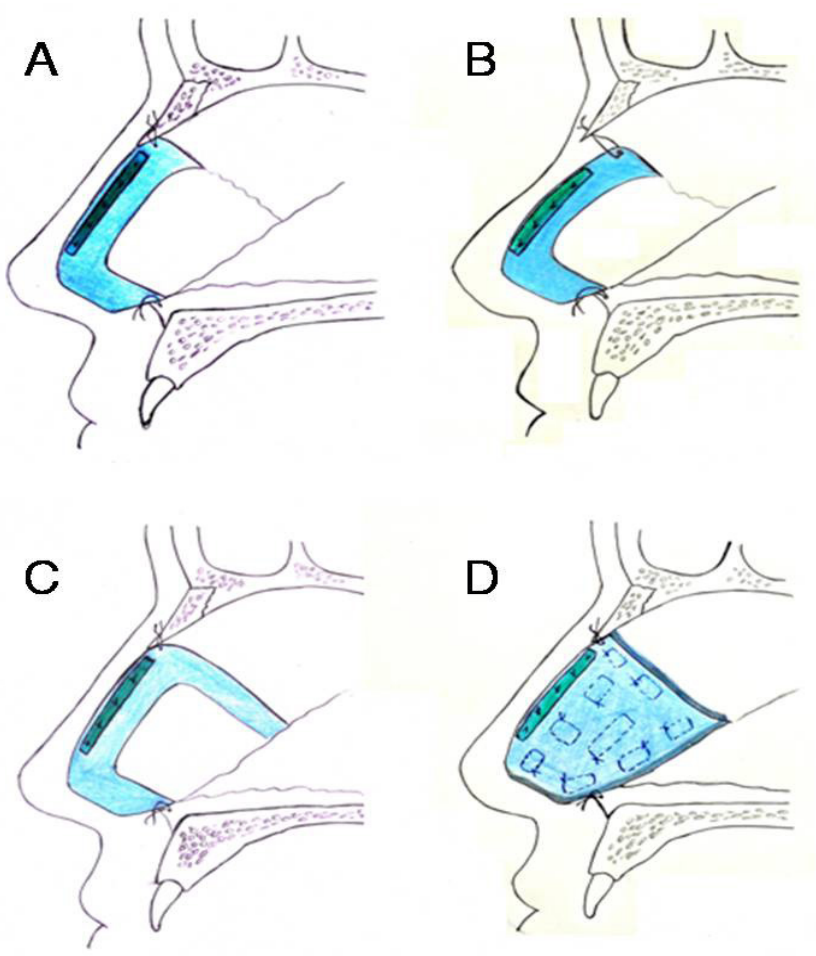

Figure 6: Schematic diagram of the technique. A: Reconstructed nasal septum with L-strut; B: collapse at the keystone area which may occur in L-strut due to inadequate suture fixation or failure of suture; C: C-shaped septal strut with two vertical limbs on both ends provides better stability; D: total septal plate replacement with costal cartilage graft. Multiple box sutures are used to hold the cartilage grafts together and prevent warping

nasal spine (ANS) caudally and nasal bone proximally, leaves no space for postoperative saddling, which is common with L-strut due to loosening or breaking of suture [Figure 6B]. Authors strongly advocate the replacement of either complete septal plate [Figure 6D] or a C-shaped septal strut [Figure 6C] with two vertical limbs on both ends (unlike L-strut which has only one vertical limb supported on ANS while the stability of cephalic end is largely dependent on suture fixation, which is not always reliable).

Replacing the complete septum is advantageous because it prevents primary sinking and collapse at the keystone area which may occur in L-strut due to inadequate suture fixation or failure of suture, secondary sinking of septum or shortening of nose due to development of fibrosis in empty space and vibration of empty mucoperichondrial flaps. ${ }^{\left[{ }^{[9]}\right.}$ It also provides a solid support to the nose, and if required later, makes the subsequent surgical dissection easier (since it is easy to separate mucochondrial flaps from cartilage than from each other).

This procedure is simple, easy to understand and reproducible. Though this is a very small series, authors 
feel that this new technique of formation of complete neo-septum with costal cartilage is very helpful when used with specific indications and provides an additional tool to the rhinoplasty surgeon to be used in cases of difficult secondary septoplasty.

\section{Financial support and sponsorship} None.

\section{Conflicts of interest}

There are no conflicts of interest.

\section{Patient consent}

Obtained.

\section{Ethics approval}

All procedures performed in studies involving human participants were in accordance with the ethical standards of the instistutional and/or national research committee and with the 1964 Helsinki declaration and its later amendments or comparable ethical standards.

\section{REFERENCES}

1. Agrawal KS, Singla S, Shrotriya R. "Text-Neck View": a new photographic tool for assessment of nasal dorsum in crooked noses. Plast Reconstr Surg 2016;138:165e-7e.

2. Agrawal KS, Bachhav M, Shrotriya R. Namaste (counterbalancing) technique: overcoming warping in costal cartilage. Indian J Plast Surg 2015;48:123-8.

3. King ED, Ashley FL. The correction of the internally and externally deviated nose. Plast Reconstr Surg 1952;10:116-20.

4. Gubisch W. The extracorporeal septum plasty: a technique to correct difficult nasal deformities. Plast Reconstr Surg 1995;95:672-82.

5. Gubisch W, Constantinescu MA. Refinements in extracorporal septoplasty. Plast Reconstr Surg 1999;104:1131-42.

6. Gubisch W. Extracorporeal septoplasty for the markedly deviated septum. Arch Facial Plast Surg 2005;7:218-26.

7. Gerlinger I, Kárász T, Somogyvári K, Szanyi I, Ráth G, Móricz P, Boenish M. Extracorporeal septal reconstruction with polydioxanone foil. Clin Otolaryngol 2007;32:465-70.

8. James SE, Kelly MH. Cartilage recycling in rhinoplasty: polydioxanone foil as an absorbable biomechanical scaffold. Plast Reconstr Surg 2008;122:254-60.

9. Boenisch M, Tamás H, Nolst Trenité GJ. Morphological and histological findings after typical surgical manipulations on growing septal cartilage in rabbits. Facial Plast Surg 2007;23:231-7. 